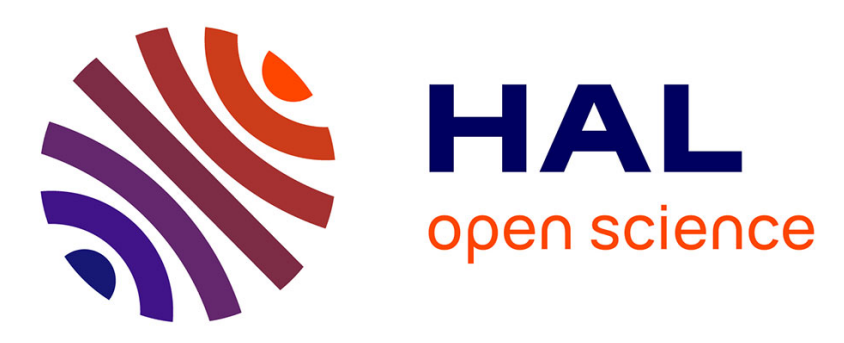

\title{
Adaptive PSK Modulation Scheme in the Presence of Phase Noise
}

Simon Bicaïs, Jean-Baptiste Doré, José Luis Gonzalez Jimenez

\section{To cite this version:}

Simon Bicaïs, Jean-Baptiste Doré, José Luis Gonzalez Jimenez. Adaptive PSK Modulation Scheme in the Presence of Phase Noise. 2018 IEEE 19th International Workshop on Signal Processing Advances in Wireless Communications (SPAWC) , Jun 2018, Kalamata, Greece. hal-01807047

\section{HAL Id: hal-01807047 \\ https://hal.science/hal-01807047}

Submitted on 4 Jun 2018

HAL is a multi-disciplinary open access archive for the deposit and dissemination of scientific research documents, whether they are published or not. The documents may come from teaching and research institutions in France or abroad, or from public or private research centers.
L'archive ouverte pluridisciplinaire HAL, est destinée au dépôt et à la diffusion de documents scientifiques de niveau recherche, publiés ou non, émanant des établissements d'enseignement et de recherche français ou étrangers, des laboratoires publics ou privés. 


\title{
Adaptive PSK Modulation Scheme in the Presence of Phase Noise
}

\author{
Simon Bicaïs ${ }^{\dagger}$, Jean-Baptiste Doré ${ }^{\dagger}$, José Luis Gonzalez Jimenez ${ }^{\dagger}$ \\ ${ }^{\dagger}$ CEA-Leti, Mintatec Campus, Grenoble, France \\ contact: simon.bicais@cea.fr
}

\begin{abstract}
Phase noise is one of the major impairments affecting severely performance of millimeter-wave systems. This paper addresses the problem of link adaption for coherent and non-coherent phase modulated signals subject to Gaussian and Wiener phase noise. We first derive closed-form approximations of the bit error rate. Then, in contrast to usual link adaptation techniques, we propose a simple scheme exploiting estimations of not only the signal-to-noise ratio but also of the phase noise variance, which is essential to achieve reliable communications.

Index Terms-Millimeter wave, Phase noise, Phase shift keying, Adaptive Systems, Performance analysis, Channel estimation
\end{abstract}

\section{INTRODUCTION}

Future 5G millimeter-wave (mmWave) bands should lead to user throughputs above $1 \mathrm{Gbit} / \mathrm{s}$. However, additional breakthrough technologies are necessary to reach this requirement. Therefore, for the first time in the telecommunication history, bands above $90 \mathrm{GHz}$ are studied for future communications [1]. In particular, mmWave systems are critically impacted by phase noise (PN) due to the poor performance of high frequency oscillators [2]. This has motivated extensive work on PN estimation and compensation [3], [4]. Further, achieving high-rate communications over wireless links in the mmWave domain demands tremendous amount of power, and so, highefficiency and wide-bandwidth power amplifiers [5]. Therefore, coherent Phase Shift Keying (PSK) modulations are highly valuable since they demonstrate a constant envelope property, and thus, offer an efficient use of power amplifiers. Yet, PSK modulations are highly sensitive to phase related impairments: Carrier Frequency Offset (CFO), PN. Differential PSK (DPSK) has been introduced as a more robust scheme. At the expense of a noise enhancement, DPSK enables noncoherent communications [6]. Prior work in [7] has confronted the robustness of PSK and DPSK when affected by a Tikohonov PN. Setting the modulation scheme to maximize spectral efficiency while maintaining robustness is correspondingly the motivation behind link adaption. Such adaptive systems are now implemented in most of practical systems [8] and rely on a channel quality estimation to set the modulation.

Contributions: In this paper, we seek to improve the design of inherently robust communication systems impacted by PN. First, we assume a high Signal-to-Noise Ratio (SNR) and a Gaussian PN to derive a closed-form approximation of the Bit Error Rate (BER) for any $M$-ary PSK. Considering that the superposition of a Gaussian and a Wiener process is a more realistic PN model [9], [10], we derive and confront the BER performance of PSK and DPSK for such PN. Furthermore, in contrast to usual link adaptation techniques, we propose a simple scheme exploiting estimations of not only the SNR but also of the PN variance, which proves to be truly essential to achieve reliable communications. We hence derive the appropriate Maximum Likelihood (ML) estimators of the thermal and phase noise variances. Eventually, we propose a statistical test to determine whether the coherent or noncoherent modulation is the most robust one and should be employed.

Organization: The remainder of this paper is structured as follows. Section II introduces a brief description of the channel and PN models. Section III is devoted to the performance analysis of PSK and DPSK impacted by Gaussian and Wiener PN. Finally, Section IV derives the appropriate channel estimation to implement the link adaptation scheme.

\section{SYSTEM MODEL}

\section{A. Channel model}

Considering a single carrier communication system with a perfectly mitigated channel, the received symbol at time instant $k$ is defined by

$$
r_{k}=s_{k} \cdot e^{j \phi_{k}}+n_{k},
$$

where $s$ is the modulated symbol from constellation $\mathcal{C}$ with average symbol energy $E_{s}, \phi$ is the oscillator $\mathrm{PN}$ and $n$ represents independent identically distributed samples of a zero-mean complex Additive White Gaussian Noise (AWGN) with variance $2 \sigma_{n}^{2}$. A PSK constellation with modulation order $M$ is defined by $\mathcal{C}=\left\{\sqrt{E_{s}} \exp \left(j \frac{2 \pi}{M} \cdot i\right) \mid i=1, \ldots, M\right\}$. In the case of a DPSK, information is encoded in the phase difference from one symbol to another denoted $\delta \theta_{k}=\left\langle s_{k}-\left\langle s_{k-1}\right.\right.$. We denote by $\angle s$ and $|s|$ the phase and amplitude of a symbol $s$.

\section{B. Phase noise model}

Oscillators PN is generated from the transformation of amplitude fluctuations into phase fluctuations [11], such that it describes a cumulative random process [9], [12]. If only white noise sources are considered ${ }^{1}$, the oscillator $\mathrm{PN} \phi$ may thus be modeled by the superposition of a Wiener (Gaussian random-walk) process $\phi_{w}$ and a Gaussian one $\phi_{g}$ [9]. This may be expressed by

$$
\begin{aligned}
\phi_{k} & =\phi_{w, k}+\phi_{g, k}, & \phi_{g, k} & \sim \mathcal{N}\left(0, \sigma_{g}^{2}\right), \\
\phi_{w, k} & =\phi_{w, k-1}+\delta \phi_{w, k}, & \delta \phi_{w, k} & \sim \mathcal{N}\left(0, \sigma_{w}^{2}\right),
\end{aligned}
$$

where $\sigma_{w}^{2}$ and $\sigma_{g}^{2}$ denote respectively the variances of the Wiener PN increment $\delta \phi_{w}$ and the Gaussian PN. Besides mathematical convenience [13], [14], the Gaussian distribution is also a relevant PN model. When considering wide bandwidth systems ${ }^{2}$, the oscillator noise floor represents the

\footnotetext{
${ }^{1}$ Flicker noise is diregarded in this study.

${ }^{2}$ Typically the case for mmWave systems
} 
greatest contribution to the overall PN [10], such that the Wiener PN becomes negligible compared to the Gaussian one, i.e. $\sigma_{g}^{2}>\sigma_{w}^{2}$. Thereby, Section III-A and IV-A use the Gaussian PN model to pursue a simple analytical analysis while Section III-B and IV-B extend it to the sum of Wiener and Gaussian PN.

\section{Performance Analysis}

\section{A. PSK over Gaussian phase noise}

To begin with, let us assume that the channel is dominated by Gaussian PN. Regarding symbol-by-symbol detection, it is known that the ML criterion minimizes the Symbol Error Probability (SEP) [6], [15]. In order to design such optimum receiver, let us derive the likelihood function, which may be rewritten as

$$
p\left(r_{k} \mid s_{k}\right)=p\left(\left|r_{k}\right|, \not r_{k}|| s_{k} \mid, \angle s_{k}\right) .
$$

By definition, the AWGN is invariant under rotation such that, for simplicity of notation, $n$ may stand for $n \cdot e^{j \theta}$ for any $\theta$ fixed. The received symbol should be studied through its amplitude and phase as

$$
\begin{gathered}
\left|r_{k}\right|=\mid\left(\left|s_{k}\right|+n_{k}\right) \cdot e^{j\left(\angle s_{k}+\phi_{k}\right) \mid} \\
=\sqrt{\left(\left|s_{k}\right|+\Re\left(n_{k}\right)\right)^{2}+\Im\left(n_{k}\right)^{2}} \\
\simeq \sqrt{E_{s}}+\Re\left(n_{k}\right) \\
\angle r_{k}=\arg \left[\left(\left|s_{k}\right|+n_{k}\right) \cdot e^{j\left(\left\lfloor s_{k}+\phi_{k}\right)\right.}\right] \\
=\angle s_{k}+\phi_{k}+\arctan \left(\frac{\Im\left(n_{k}\right)}{\left|s_{k}\right|+\Re\left(n_{k}\right)}\right) \\
\simeq \angle s_{k}+\phi_{k}+\frac{\Im\left(n_{k}\right)}{\sqrt{E_{s}}} .
\end{gathered}
$$

These first-order approximations are tight for a high SNR scenario [16], [17]. By Eq. (2), the likelihood function follows a bivariate Gaussian distribution and is thus expressed by

$$
p\left(r_{k} \mid s_{k}\right)=\frac{\exp \left(-\frac{1}{2}\left(\frac{\left(\left|r_{k}\right|-\sqrt{E_{s}}\right)^{2}}{\sigma_{n}^{2}}+\frac{\left(\not r_{k}\right.}{\sigma_{g}^{2}+\sigma_{n}^{2} / E_{s}}\right)^{2}\right.}{2 \pi \sqrt{\sigma_{n}^{2}\left(\sigma_{g}^{2}+\sigma_{n}^{2} / E_{s}\right)}} .
$$

This expression, originally derived in [14], can be exploited in our study to approximate the BER of a PSK. With respect to the ML decision rule for a PSK, the detected symbol is

$$
\begin{aligned}
\hat{s_{k}} & =\underset{s \in \mathcal{C}}{\operatorname{argmax}} p\left(r_{k} \mid s_{k}\right) \\
& =\underset{s \in \mathcal{C}}{\operatorname{argmin}}\left(\angle r_{k}-\angle s_{k}\right)^{2},
\end{aligned}
$$

which enables to derive the SEP as follows

$$
\begin{aligned}
& P_{s e}=1-\operatorname{Pr}\left(\hat{s_{k}}=s_{k} \mid s_{k}\right) \\
& =1-p\left(-\frac{\pi}{M}<\angle r_{k}-\angle s_{k}<\frac{\pi}{M}\right) \\
& =2 Q\left(\frac{\pi}{M \sqrt{\sigma_{g}^{2}+\sigma_{n}^{2} / E_{s}}}\right) \text {. }
\end{aligned}
$$

To directly relate the BER to the SEP, we shall make two commonly used assumptions. First, the bit labeling of the constellation satisfies a Gray coding. Furthermore, misdetections

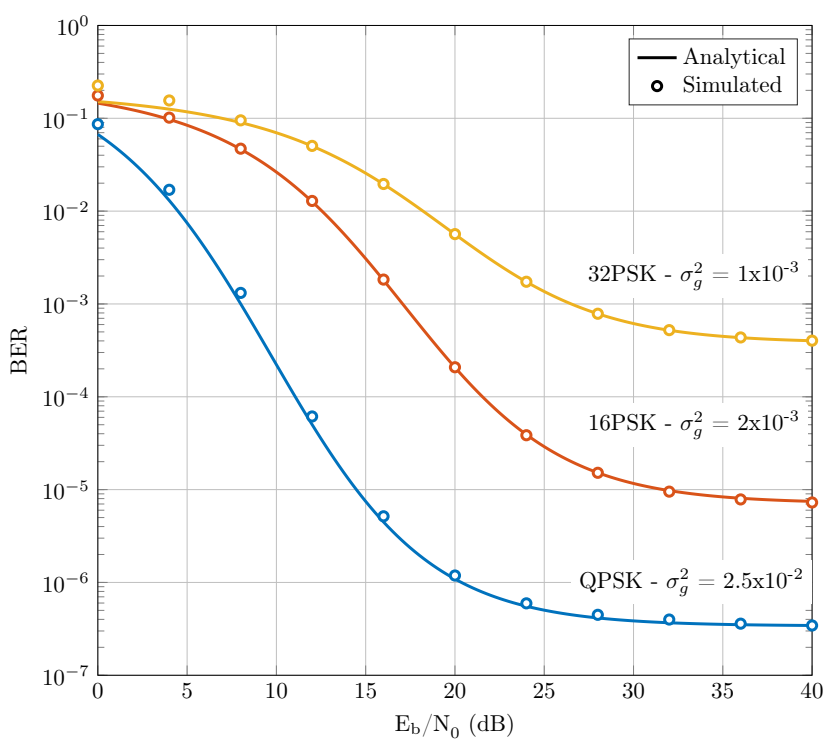

Fig. 1: Analytical and simulated BER performance of $M$-ary PSK affected by Gaussian PN with variance $\sigma_{g}^{2}$

only occur on the nearest neighbors of the sent symbol. Finally, the BER of a PSK affected by Gaussian PN is approximated by

$$
P_{b e, \mathrm{PSK}}^{g} \simeq \frac{2}{\log _{2}(M)} Q\left(\frac{\pi}{M \sqrt{\sigma_{g}^{2}+\frac{1}{2 \cdot \log _{2}(M)} \frac{1}{E_{b} / N_{0}}}}\right),
$$

where the average bit energy $E_{b}$ satisfies $E_{s}=E_{b} \log _{2}(M)$ and the noise power spectral density is defined by $N_{0}=2 \sigma_{n}^{2}$. The BER performance of PSK for arbitrary orders of modulation and PN variances are illustrated in Fig. 1. As exhibited, the closed-form expression holds accurate approximations of the BER at high SNR.

\section{B. DPSK over Wiener phase noise}

DPSK enables a non-coherent demodulation at the receiver. As a consequence, estimation of the carrier phase is not required. Moreover, a differential modulation is not subject to the cumulative nature of the PN, and only slightly to the CFO. This robustness is achieved at the expense of a BER performance degradation. Yet, the stronger these impairments, the more valuable it seems to opt for differential encoding. The pending question is when DPSK is more robust than PSK. Therefore, let us derive the performance of PSK and DPSK when the PN is described by the sum of a Wiener and a Gaussian PN. The optimum decision for a DPSK receiver [6] is given by

$$
\hat{\delta \theta}=\underset{\delta \theta \in \mathcal{C}}{\operatorname{argmin}}\left(\left(\measuredangle r_{k}-\angle r_{k-1}\right)-\delta \theta\right)^{2} .
$$

With regard to the PN model in Eq. (2) and to the high SNR approximation in Eq. (5), we have

$\angle r_{k}-\angle r_{k-1} \simeq \delta \theta_{k}+\delta \phi_{w, k}+\phi_{g, k}-\phi_{g, k-1}+\frac{\Im\left(n_{k}-n_{k-1}\right)}{\sqrt{E s}}$, 


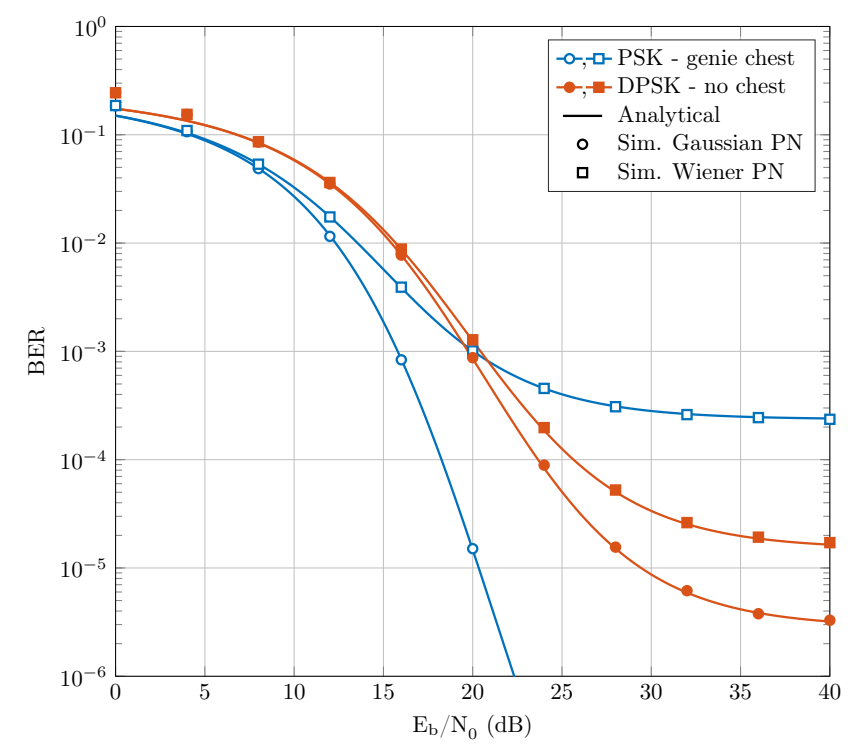

Fig. 2: Analytical and simulated BER performance of 16PSK and 16DPSK for Gaussian PN $\left(\sigma_{g}^{2}=10^{-3}, \sigma_{w}^{2}=0\right)$ and Wiener PN $\left(\sigma_{g}^{2}=10^{-3}, \sigma_{w}^{2}=\sigma_{g}^{2} / 2\right)$

which is directly equivalent to

$$
\begin{gathered}
\angle r_{k}-\angle r_{k-1} \simeq \delta \theta_{k}+\phi_{e q, k}, \\
\text { where } \phi_{e q} \sim \mathcal{N}\left(0, \sigma_{w}^{2}+2 \sigma_{g}^{2}+2 \sigma_{n}^{2} / E_{s}\right) .
\end{gathered}
$$

Thereupon, the BER for a DPSK subject to Gaussian and Wiener PN may be approximated by

$$
P_{b e, \mathrm{DPSK}}^{w} \simeq \frac{2}{\log _{2}(M)} Q\left(\frac{\pi}{M \sqrt{\sigma_{w}^{2}+2 \sigma_{g}^{2}+\frac{1}{E_{b} / N_{0}} \frac{1}{\log _{2}(M)}}}\right) .
$$

To establish a comparison with the PSK modulation, we consider a genie estimation at the PSK receiver. With one pilot symbol, the receiver is able to estimate and compensate perfectly the Wiener process. The pilot period is defined by $T$, such that if $k=n \cdot T$ then $\phi_{w, k+1}=\delta \phi_{w, k}$. The estimation problem in presence of Wiener PN exceeds the scope of this paper, but is addressed in [4]. Though the derivation is not developed here, the BER for a PSK in this case is given by

$$
\begin{aligned}
P_{b e, \mathrm{PSK}}^{w} & \simeq \frac{1}{T-1} \sum_{k=1}^{T-1} \frac{2}{\log _{2}(M)} \\
\cdot & Q\left(\frac{\pi}{M \sqrt{k \cdot \sigma_{w}^{2}+\sigma_{g}^{2}+\frac{T / T-1)}{E_{b} / N_{0}} \frac{1}{2 \cdot \log _{2}(M)}}}\right) .
\end{aligned}
$$

Figure 2 confronts the performance of a 16PSK to the ones of a 16DPSK for Gaussian and Wiener PN. The PSK receiver presents a pilot density of $10 \%$ with a genie estimation. Conversely, the DPSK receiver does not implement any channel estimation. Figure 2 shows that analytical expressions are tight to the simulation results. Moreover, Fig. 2 confirms an aforementioned intuition. The DPSK presents a loss in BER performance, still, when considering stronger Wiener PN then it is more robust than the PSK.

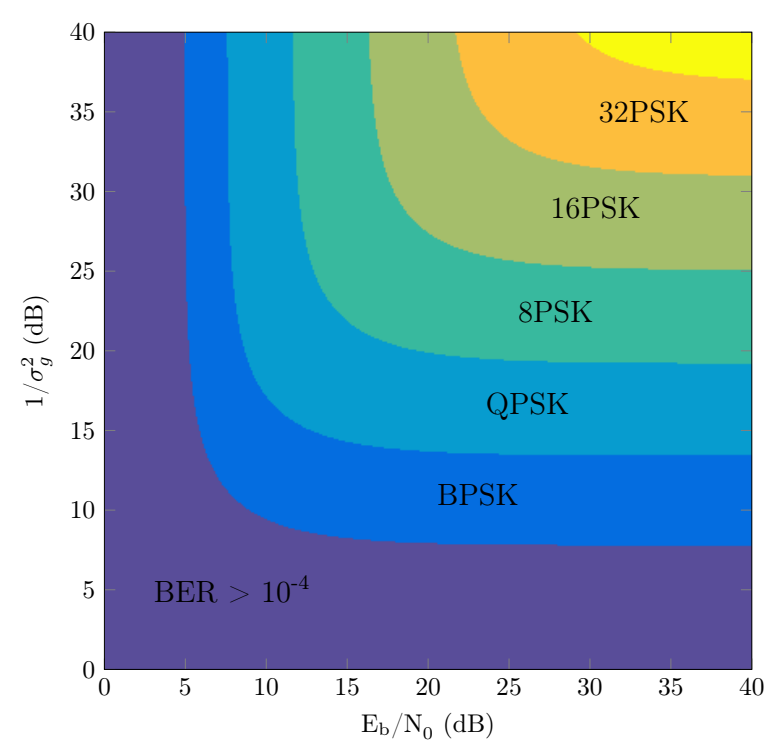

Fig. 3: Modulation Scheme Regions for $M$-ary PSK: highest modulation order $M$ achieving BER $<10^{-4}$ as a function of $E_{b} / N_{0}$ and the inverse of PN variance $1 / \sigma_{g}^{2}$

\section{LINK ADAPTION}

\section{A. Estimation of thermal and phase noise variances}

To improve spectral efficiency, practical communication systems implement adaptive modulation scheme [8]. Namely, the modulation order is inferred from a SNR estimation. In the case of a channel dominated by Gaussian PN, the analytical approximation of the BER in Eq. (9) enables to determine the greatest value of $M$ while maintaining the error rate below a fixed target. Figure 3 depicts the modulation scheme regions for $M$-ary PSK, i.e. the highest modulation order $M$ achieving BER $<10^{-4}$ given $E_{b} / N_{0}$ and $\sigma_{g}^{2}$. It is shown that the performance is highly related to the PN variance, which should necessarily be considered in the link adaptation to maintain robustness.

Such link adaptation requires an estimation of $\sigma_{n}^{2}$ and $\sigma_{g}^{2}$. This may be realized by inserting $N$ pilot samples possibly distributed and known from the receiver denoted $\boldsymbol{s}=\left(s_{1}, s_{2}, \ldots, s_{N}\right)$ and by deriving the appropriate estimators. From Eq. (6), the joint likelihood function of the $N$ received samples $\boldsymbol{r}=\left(r_{1}, \ldots, r_{N}\right)$ is given by

$$
p_{N}\left(\boldsymbol{r} \mid \boldsymbol{s}, \sigma_{n}^{2}, \sigma_{g}^{2}\right)=\prod_{k=1}^{N} p\left(r_{k} \mid s_{k}, \sigma_{n}^{2}, \sigma_{g}^{2}\right) .
$$

We derive the ML estimate of the covariance matrix for the multivariate normal distribution [15]. It yields here the ML estimators of $\sigma_{n}^{2}$ and $\sigma_{g}^{2}$ expressed by

$$
\begin{aligned}
& \hat{\sigma}_{n}^{2}=\frac{1}{N} \sum_{k=1}^{N}\left(\left|r_{k}\right|-\sqrt{E_{s}}\right)^{2}, \\
& \hat{\sigma}_{g}^{2}=\frac{1}{N} \sum_{k=1}^{N}\left(\angle r_{k}-\angle s_{k}\right)^{2}-\frac{\hat{\sigma}_{n}^{2}}{E_{s}} .
\end{aligned}
$$

Writing these estimators as $\chi^{2}(N)$ distributions enables us to quantify their performance in terms of bias and dispersion 
respectively with the means and variances given by

$$
\left\{\begin{array}{l}
\mathrm{E}\left[\hat{\sigma}_{n}^{2}\right]=\sigma_{n}^{2} \\
\mathrm{~V}\left[\hat{\sigma}_{n}^{2}\right]=\frac{2 \sigma_{n}^{4}}{N}
\end{array}, \quad\left\{\begin{array}{l}
\mathrm{E}\left[\hat{\sigma}_{g}^{2}\right]=\sigma_{g}^{2} \\
\mathrm{~V}\left[\hat{\sigma}_{g}^{2}\right]=\frac{2\left(\sigma_{g}^{2}+\sigma_{n}^{2} / E_{s}\right)^{2}}{N}+\frac{2 \sigma_{n}^{4}}{N E_{s}}
\end{array} .\right.\right.
$$
(17)

Since these estimators are unbiased and the regularity conditions clearly satisfied in Eq. (6), we may compare the estimators to their Cramer-Rao Lower Bounds (CRLB) [18] written as

$$
\mathrm{V}\left[\hat{\sigma}_{n}^{2}\right] \geq \frac{2 \sigma_{n}^{4}}{N}, \quad \mathrm{~V}\left[\hat{\sigma}_{g}^{2}\right] \geq \frac{2\left(\sigma_{g}^{2}+\sigma_{n}^{2} / E_{s}\right)^{2}}{N} .
$$

It follows easily that the thermal noise variance estimator is efficient in the sense of achieving the CRLB $c f$. [15]. As for the PN variance, the estimator is tight to the CRLB but does not reach it. However, both estimators demonstrate the smallest Mean Square Error (MSE) among any unbiased estimators. From Eq. (15), it is straightforward that the joint density $p_{N}$ belongs to the exponential family such that $S=\left(\sum_{k=1}^{N}\left(\left|r_{k}\right|-\sqrt{E_{s}}\right)^{2}, \sum_{k=1}^{N}\left(/ r_{k}-/ s_{k}\right)^{2}\right)$ is a complete sufficient statistic for parameter $\left(\sigma_{n}^{2}, \sigma_{g}^{2}\right)$. By the Lehman-Scheffé theorem, the unbiased estimators $\left(\hat{\sigma}_{n}^{2}, \hat{\sigma}_{g}^{2}\right)$, defined upon $S$, are respectively the unique MinimumVariance Unbiased Estimators (MVUE) of $\sigma_{n}^{2}$ and $\sigma_{g}^{2}$.

\section{B. Is the phase noise a cumulative process?}

As illustrated in Fig. 2, the DPSK should be favored at the detriment of PSK only if the cumulative nature of the PN is non-negligible. Hence, the link adaptation problem can be expressed as a simple detection problem: is the cumulative process of the phase noise strong enough to advantage a differential modulation. Let us formulate the corresponding binary hypothesis test as

$$
\begin{array}{lr}
H_{0}: \text { Gaussian } \mathrm{PN}, & \text { if } \sigma_{w}^{2} / \sigma_{g}^{2}<\lambda \text { fixed, } \\
H_{1} \text { : Wiener PN, } & \text { otherwise. }
\end{array}
$$

The link adaptation decision $\delta$ is then expressed by

$$
\delta(\boldsymbol{r})=\left\{\begin{array}{ll}
\mathrm{PSK}, & \text { if } S(\boldsymbol{r})<\varphi(\lambda) \\
\mathrm{DPSK}, & \text { otherwise }
\end{array},\right.
$$

where $\boldsymbol{r}=\left(r_{1}, \ldots, r_{N}\right)$ is a received sequence of $N$ consecutive pilots, $S$ denotes a sufficient statistic of the received pilots sample and $\varphi$ is a test threshold function of $\lambda$. Fortuitously, this problem has been extensively studied in the financial field under a different name: the random walk hypothesis for stock market prices [19]. Numerous statistical tests have been designed, yet, one in particular has retained most of the attention: the variance ratio test [19], [20]. This test relies on the fact that the variance of the random walk increments increases linearly with $p$ the sampling interval. Applied to our context, this is expressed by

$$
V\left[\phi_{k+p}-\phi_{k}\right]=p \cdot \sigma_{w}^{2}+2 \sigma_{g}^{2},
$$

which is denoted further by $\sigma_{\Delta p}^{2}$. Letting $\sigma_{w}^{2}=\lambda \cdot \sigma_{g}^{2}$ and summing over $p$, the statistical test becomes

$$
S(\boldsymbol{r})=\sum_{p=1}^{M} \frac{\hat{\sigma}_{\Delta p}^{2}}{\hat{\sigma}_{\Delta 1}^{2}}<\varphi(\lambda)=\frac{\lambda \cdot M(M+1)+4 M}{4 \lambda+2},
$$

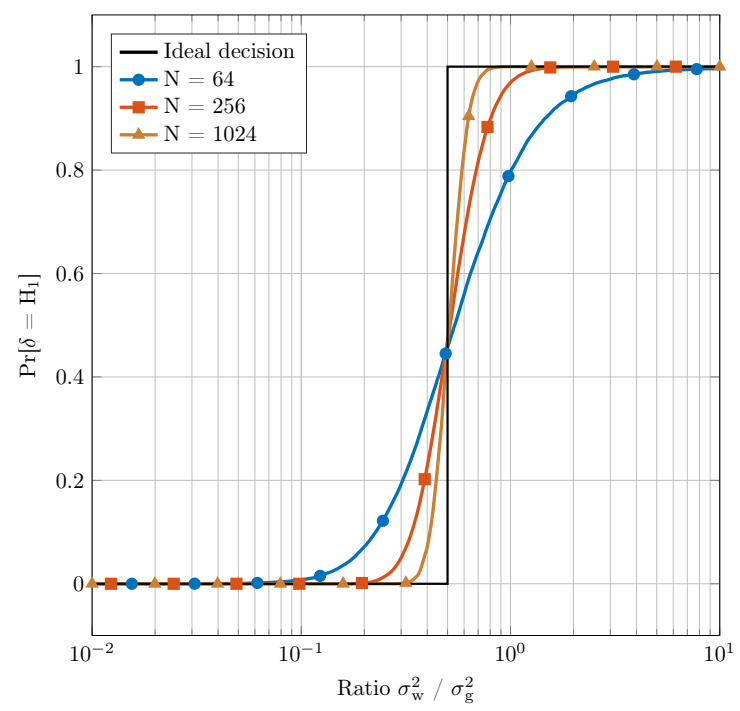

Fig. 4: Mean probability of taking decision $\delta(\boldsymbol{r})=H_{1}$ when $\lambda=1 / 2$ for different lengths of pilot sequence $N$

where $\hat{\sigma}_{\Delta p}^{2}$ is the estimator of $\sigma_{\Delta p}^{2}$ and evaluated by

$$
\hat{\sigma}_{\Delta p}^{2}=\frac{1}{N-p} \sum_{k=1}^{N-p}\left(\left(\angle r_{k+p}-\angle s_{k+p}\right)-\left(\angle r_{k}-\angle s_{k}\right)\right)^{2} .
$$

The upper bound of summation $M$ satisfies $1<M<N$, and is optimized heuristically ${ }^{3}$. Figure 4 depicts the mean probability of taking decision $\delta(\boldsymbol{r})=H_{1}$ and thus use DPSK. Several pilot lengths are considered and $\lambda$ is set to $1 / 2$ as in Fig. 2 . As exhibited, the longer the pilot sequence, the more accurate the test. The performance degradation caused by an incorrect decision should be evaluated to properly specify the length of the training sequence. Due to space limitation, it is not carried out here but using the proposed framework, it is straightforward to derive.

\section{CONCLUSION}

In this paper, we have addressed the problematic of link adaption for phase modulated signals affected by PN. We have first considered PSK modulations impacted by a Gaussian $\mathrm{PN}$ and provided the closed-form approximation of the BER. Optimum ML estimators of thermal and phase noise variances have been derived to propose a link adaptation scheme. Exploiting estimations of both the SNR and the PN variance proved to be essential to maintain robustness. Then, the PN has been described with a more realistic model: a sum of a Gaussian and a Wiener process. Performance of PSK and DPSK were compared in order to determine when the use of a differential modulation is beneficial. Accordingly, we proposed a simple detection test, inspired from the financial field, to perform the link adaptation and exploit the most robust modulation between PSK and DPSK. Nevertheless, these results remain an analytical analysis and it is of particular interest to investigate this adaptation problem under practical implementation considerations.

\footnotetext{
${ }^{3}$ The upper bound $M$ in Eq. 22 must be large enough to yield a robust test. However, as the sampling interval $p$ gets bigger, the dispersion of $\hat{\sigma}_{\Delta p}^{2}$ in Eq. 23 increases. Thus, if $M$ is too large, the test is defined upon unreliable estimations.
} 


\section{ACKNOWLEDGMENT}

The research leading to these results received funding from the French National Research Agency (ANR-17-CE25-0013) within the frame of the project BRAVE.

\section{REFERENCES}

[1] G. Fettweis, F. Guderian, and S. Krone, "Entering the path towards terabit/s wireless links," in 2011 Design, Automation Test in Europe, March 2011, pp. 1-6.

[2] M. Voicu, D. Pepe, and D. Zito, "Performance and trends in millimetrewave cmos oscillators for emerging wireless applications," International Journal of Microwave Science and Technology, vol. 2013, p. 6, 2013.

[3] X. Yi, W. Shieh, and Y. Tang, "Phase estimation for coherent optical ofdm transmission," in COIN-ACOFT 2007 - Joint International Conference on the Optical Internet and the 32nd Australian Conference on Optical Fibre Technology, June 2007, pp. 1-3.

[4] F. Munier, E. Alpman, T. Eriksson, A. Svensson, and H. Zirath, "Estimation of phase noise for qpsk modulation over awgn channels," in GigaHertz 2003. Proceedings from the Seventh Symposium, no. 8. Linkping University Electronic Press; Linkpings universitet, 2003.

[5] H. J. Song and T. Nagatsuma, "Present and future of terahertz communications," IEEE Transactions on Terahertz Science and Technology, vol. 1, no. 1, pp. 256-263, Sept 2011.

[6] J. Proakis, Digital Communications, ser. McGraw-Hill series in electrical and computer engineering : communications and signal processing. McGraw-Hill, 2001.

[7] A. G. Burr, "Comparison of coherent and noncoherent modulation in the presence of phase noise," IEE Proceedings I - Communications, Speech and Vision, vol. 139, no. 2, pp. 147-155, April 1992.

[8] A. Technologies and M. Rumney, LTE and the Evolution to $4 G$ Wireless: Design and Measurement Challenges, 2nd ed. Wiley Publishing, 2013

[9] A. Demir, "Computing timing jitter from phase noise spectra for oscillators and phase-locked loops with white and1/fnoise," IEEE Transactions on Circuits and Systems I: Regular Papers, vol. 53, no. 9, pp. 1869-1884, Sept 2006.

[10] M. R. Khanzadi, D. Kuylenstierna, A. Panahi, T. Eriksson, and H. Zirath, "Calculation of the performance of communication systems from measured oscillator phase noise," IEEE Transactions on Circuits and Systems I: Regular Papers, vol. 61, no. 5, pp. 1553-1565, May 2014.

[11] A. Chorti and M. Brookes, "A Spectral Model for RF Oscillators With Power-Law Phase Noise," IEEE Transactions on Circuits and Systems I: Regular Papers, vol. 53, no. 9, pp. 1989-1999, Sept 2006.

[12] A. Hajimiri and T. H. Lee, "A general theory of phase noise in electrical oscillators," IEEE Journal of Solid-State Circuits, vol. 33, no. 2, pp. 179-194, Feb 1998.

[13] M. R. Khanzadi, H. Mehrpouyan, E. Alpman, T. Svensson, D. Kuylenstierna, and T. Eriksson, "On models, bounds, and estimation algorithms for time-varying phase noise," in 2011 5th International Conference on Signal Processing and Communication Systems (ICSPCS), Dec 2011, pp. $1-8$.

[14] R. Krishnan, A. G. i Amat, T. Eriksson, and G. Colavolpe, "Constellation optimization in the presence of strong phase noise," IEEE Transactions on Communications, vol. 61, no. 12, pp. 5056-5066, December 2013.

[15] T. Moon and W. Stirling, Mathematical Methods and Algorithms for Signal Processing. Prentice Hall, 2000.

[16] H. Fu and P. Y. Kam, "Exact phase noise model and its application to linear minimum variance estimation of frequency and phase of a noisy sinusoid," in 2008 IEEE 19th International Symposium on Personal, Indoor and Mobile Radio Communications, Sept 2008, pp. 1-5.

[17] R. Krishnan, M. R. Khanzadi, T. Eriksson, and T. Svensson, "Soft metrics and their performance analysis for optimal data detection in the presence of strong oscillator phase noise," CoRR, vol. abs/1310.1638, 2013.

[18] S. M. Kay, Fundamentals of Statistical Signal Processing: Estimation Theory. Upper Saddle River, NJ, USA: Prentice-Hall, Inc., 1993.

[19] A. Charles and O. Darné, "Variance ratio tests of random walk: An overview," Journal of Economic Surveys, vol. 23, no. 3, pp. 503-527, 2009.

[20] A. W. Lo and A. C. MacKinlay, "Stock market prices do not follow random walks: Evidence from a simple specification test," The Review of Financial Studies, vol. 1, no. 1, pp. 41-66, 1988. 